YITP-00-59

\title{
Numerical Study of Length Spectra and Low-lying Eigenvalue Spectra of Compact Hyperbolic 3-manifolds
}

\author{
Kaiki Taro Inoue \\ Yukawa Institute for Theoretical Physics, Kyoto University, Kyoto 606-8502, Japan
}

(November 11, 2018)

\begin{abstract}
In this paper, we numerically investigate the length spectra and the low-lying eigenvalue spectra of the Laplace-Beltrami operator for a large number of small compact(closed) hyperbolic $(\mathrm{CH}) 3$ manifolds. The first non-zero eigenvalues have been successfully computed using the periodic orbit sum method, which are compared with various geometric quantities such as volume, diameter and length of the shortest periodic geodesic of the manifolds. The deviation of low-lying eigenvalue spectra of manifolds converging to a cusped hyperbolic manifold from the asymptotic distribution has been measured by $\zeta-$ function and spectral distance.
\end{abstract}

\section{INTRODUCTION}

Eigenmodes of the Laplace-Beltrami operator $\Delta$ on a Riemannian manifold (or orbifold) which carry information of both local geometry and global topology

$$
(\Delta+E) u_{E}=0
$$

play a significant role in various kinds of physical systems.

In the cosmological perturbation theory, one can interpret $E^{1 / 2}$ as the wavenumber $k$ of an eigenfunction $u_{k}$ which characterises the scale of the metric or matter perturbation. In order to distinguish models with equivalent local geometry but non-equivalent global topology, it is crucial to study the behavior of low-lying eigenmodes. For spatially compact models, the lowest non-zero wavenumber $k_{1}$ (the first "excited" state) determines the maximum fluctuation scale of the perturbation since fluctuations on scale less than $k_{1}^{-1}$ are strongly suppressed. In compact flat 3-manifolds, $k_{1}^{-1}$ is approximately equal to an inverse of the diameter(the maximum distance between two points) of the space. Therefore, if the space elongates in one dimension and shrinks in the remaining dimension keeping the volume constant, $k_{1}$ converges to zero. For compact hyperbolic $(\mathrm{CH}) 2$-manifolds where one can deform the space continuously (without topology change) Rayleight's theorem implies that $k_{1}$ can be arbitrarily close to zero [1]. However, subtlety arises in the case of CH 3-manifolds. Because of the Mostow's rigidity theorem, one cannot deform the space continuously. Instead, we have a series of manifolds with different topology converging to a cusped manifold with finite volume (co-finite manifold). For CH 3-manifolds $M$, orthonormal basis of $L^{2}(M)$ may include supercurvarture modes $k<1$ whereas there are not any such modes in the simply-connected hyperbolic 3 -space $\mathbb{H}^{3}$. If any supercurvature modes were present, the fluctuation property on large scales would be drastically altered.

In quantum mechanics, $u_{E}$ can be interpreted as a wave function of a free particle at a stationary state with energy $E$. The statistical property of the energy eigenvalue $E$ and the eigenfunction $u_{E}$ have been investigated for exploring the imprints of classical chaos in the corresponding quantum system(e.g. see [2] and other articles therein). Because any classical dynamical systems of a free particle in $\mathrm{CH}$ spaces are strongly chaotic (K-systems), the semiclassical behavior of the statistical property of eigenvalues and eigenfunctions in these spaces has been intensively studied [3 10]. It is the Gutzwiller trace formula [11] that relates a set of periodic orbits(=geodesics) to a set of energy eigenstates and gives the semiclassical correspondence for classically chaotic systems. Interestingly, for $\mathrm{CH}$ spaces, the Gutzwiller trace formula gives an exact relation which had been known as the Selberg trace formula in mathematical literature [12]. The trace formula gives an alternative method to compute the eigenvalues in terms of periodic orbits. The poles of energy Green's function are generated as a result of interference of waves each one of which corresponds to a periodic orbit. Roughly speaking, periodic orbits with shorter length contribute to the deviation from the asymptotic eigenvalue distribution on larger energy scales. In fact, zero-length orbits produce Weyl's asymptotic formula. Because periodic orbits can be obtained algebraically, the periodic orbit sum method enables one to compute low-lying eigenvalues for a large sample of manifolds or orbifolds systematically if each fundamental group is known beforehand. The method has been used to obtain eigenvalues of the Laplace-Beltrami operator on $\mathrm{CH}$ 2-spaces and a non-arithmetic 3-orbifold [4.6. 10]. However, it has not been applied to any $\mathrm{CH} 3$-manifolds so far.

To date, various numerical techniques have been applied to the eigenvalue problems for solving the Helmholtz equation (11) with periodic boundary conditions (manifold case), Neumann and Dirichlet boundary conditions(orbifold 
case). Eigenvalues of $\mathrm{CH}$ 2-spaces has been numerically obtained by many authors [3 [5, 7, 8, 13, Eigenvalues of cusped arithmetic and cusped non-arithmetic 3-manifolds with finite volume have been obtained by Grunewald and Huntebrinker using a finite element method [14]. Aurich and Marklof have computed eigenvalues of a non-arithmetic 3 -orbifold using the direct boundary element method(DBEM) [10]. To date, computation of eigenvalue spectra is limited to a small number of 3-manifolds. The author has succeeded in computing eigenvalues of the Thurston manifold, the second smallest one using the DBEM [15], and later the Weeks manifold, the smallest one in the known $\mathrm{CH}$ manifolds using the same method [16]. Cornish and Spergel have also succeeded in calculating eigenvalues of these manifolds and 10 other $\mathrm{CH}$ manifolds based on the Trefftz method [17].

In this paper, we study the length spectra and the low-lying eigenvalue spectra of the Laplace-Beltrami operator on a relatively large number of small CH manifolds which are obtained by a computer program "SnapPea" by Weeks [18]. We analyse the fluctuating property of length spectra and check the accuracy of the first non-zero eigenvalues obtained by the periodic orbit sum method based on the trace formula. We also relate the low-lying eigenvalues to diameter, volume and length of the shortest periodic orbit and characterise the deviation of the spectrum from the asymptotic distribution. In Sec. II we briefly describe fundamental aspects of CH 3-manifolds which we will study. In Sec. III we study the length spectra of $\mathrm{CH}$ 3-manifolds, especially we put an emphasis on their fluctuating property. In Sec. IV we derive an explicit form for computing the spectral staircase in terms of length spectra from the trace formula. In Sec. V we analyse the relation between the low- lying eigenvalues and several diffeomorphism-invariant geometric quantities, namely, volume, diameter and length of the shortest periodic orbit. In Sec. VI the deviation of the low-lying eigenvalue spectra from the asymptotic distribution for manifolds that have a region similar to the neighbourhood of a cusped point is measured by $\zeta$ - function and the spectral distance.

\section{HYPERBOLIC MANIFOLDS}

The discrete subgroup $\Gamma$ of $P S L(2, \mathbb{C})$ which is the orientation-preserving isometry group of the simply-connected hyperbolic 3-space $\mathbb{H}^{3}$ is called the Kleinian group. Any $\mathrm{CH} 3$-spaces (either manifold or orbifold) can be described as compact quotients $\mathcal{M}=\mathbb{H}^{3} / \Gamma$. If we represent $\mathbb{H}^{3}$ as an upper half space $\left(x_{1}, x_{2}, x_{3}\right)$, the metric is written as

$$
d s^{2}=\frac{R^{2}\left(d x_{1}^{2}+d x_{2}^{2}+d x_{3}^{2}\right)}{x_{3}^{2}},
$$

where $R$ is the curvature radius. In what follows, $R$ is set to unity without loss of generality. If we represent a point $p$ on the upper-half space, as a quaternion whose fourth component equals zero, then the actions of $P S L(2, \mathbb{C})$ on $\mathbb{H}^{3} \cup \mathbb{C} \cup\{\infty\}$ take the form

$$
\gamma: p \rightarrow p^{\prime}=\frac{a p+b}{c p+d}, \quad a d-b c=1, \quad p \equiv z+x_{3} \mathbf{j}, \quad z=x_{1}+x_{2} \mathbf{i},
$$

where $\mathrm{a}, \mathrm{b}, \mathrm{c}$ and $\mathrm{d}$ are complex numbers and $1, \mathbf{i}$ and $\mathbf{j}$ are represented by matrices,

$$
1=\left(\begin{array}{ll}
1 & 0 \\
0 & 1
\end{array}\right), \quad \mathbf{i}=\left(\begin{array}{cc}
i & 0 \\
0 & -i
\end{array}\right), \quad \mathbf{j}=\left(\begin{array}{cc}
0 & 1 \\
-1 & 0
\end{array}\right)
$$

The action $\gamma$ is explicitly written as

$$
\begin{aligned}
\gamma: \mathbb{H}^{3} \cup \mathbb{C} \cup\{\infty\} & \rightarrow \mathbb{H}^{3} \cup \mathbb{C} \cup\{\infty\} \\
\gamma:\left(z\left(x_{1}, x_{2}\right), x_{3}\right) & \rightarrow\left(\frac{(a z+b)(\overline{c z+d})+a \bar{c} x_{3}^{2}}{|c z+d|^{2}+|c|^{2} x_{3}^{2}}, \frac{x_{3}}{|c z+d|^{2}+|c|^{2} x_{3}^{2}}\right),
\end{aligned}
$$

where a bar denotes a complex conjugate. Elements of $\Gamma$ for orientable $\mathrm{CH}$ manifolds are conjugate to

$$
\pm\left(\begin{array}{cc}
\exp (l / 2+i \phi / 2) & 0 \\
0 & \exp (-l / 2-i \phi / 2)
\end{array}\right)
$$

which are called loxodromic if $\phi \neq 0$ and hyperbolic if $\phi=0$.

Topological construction of $\mathrm{CH}$ manifolds starts with a cusped manifold with finite volume $M_{c}$ obtained by gluing ideal tetrahedra. Let us consider the case where $M_{c}$ is topologically equivalent to the complement of a knot $K$ or 


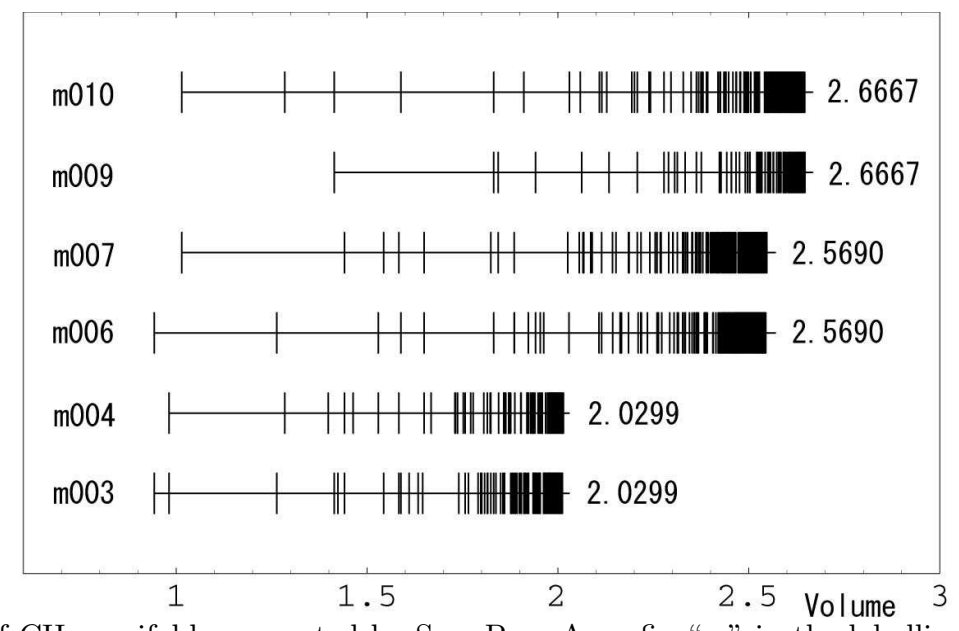

FIG. 1. Volume spectra of CH manifolds computed by SnapPea. A prefix " $m$ " in the labelling number represents a cusped manifold obtained by gluing five or fewer ideal tetrahedra. The numbers in the right side denote the volumes of the corresponding cusped manifold.

link $L$ (which consists of knots) in 3-sphere $\mathbb{S}^{3}$ or some other closed 3-spaces. A surgery in which one removes the tubular neighborhood $N$ of $K$ whose boundary is homeomorphic to a torus, and replace $N$ by a solid torus so that a meridian [ in the solid torus goes to $(p, q)$ curve ${ }^{2}$ on $N$ is called $(p, q)$ Dehn surgery. Except for a finite number of cases, Dehn surgeries on $K$ always yield $\mathrm{CH} 3$-manifolds which implies that most compact 3-manifolds are hyperbolic [19]. SnapPea can perform Dehn surgeries which have made it possible to construct a large number of samples of $\mathrm{CH}$ 3-manifolds.

It is known that only a finite number of $\mathrm{CH} 3$-manifolds with the same volume exist [19]. Hence the volume plays a crucial role in describing $\mathrm{CH}$ 3-manifolds. The key facts are: the volumes of $\mathrm{CH}$ 3-manifolds obtained by Dehn surgeries on a cusped manifold $M_{c}$ are always less than the volume of $M_{c}$; CH 3-manifolds converge to $M_{c}$ in the limit $|p|,|q| \rightarrow \infty$. As shown in figure 1, the volume spectra are discrete but there are many accumulation points which correspond to the volumes of cusped manifolds. The smallest cusped manifolds in the known manifolds have volume 2.0299 which are labeled as "m003" and "m004" in SnapPea. m003 and m004 are topologically equivalent to the complement of a certain knot in the lens space $L_{5,1}$ and the complement of a "figure eight knot" (figure 2), respectively [20]. $(3,-1)$ and $(-2,3)$ Dehn surgeries on m003 yield the smallest and the second smallest known manifolds, which are called the Weeks manifold (volume $=0.9427$ ) and the Thurston manifold (volume $=0.9814$ ), respectively. As $|p|$ and $|q|$ becomes large, the volumes converge to that of $M_{c}$. Similarly, one can do Dehn surgeries on m004 or other cusped manifolds to obtain a different series of $\mathrm{CH}$ manifolds.

\section{LENGTH SPECTRA}

Computation of periodic orbits (geodesics) are of crucial importance for the semiclassical quantization of classically chaotic systems which will be discussed in the next section. However, in general, solving a large number of periodic orbits often becomes an intractable problem since the number of periodic orbits grows exponentially with an increase in length. For $\mathrm{CH}$ manifolds periodic orbits can be calculated algebraically since each periodic orbit corresponds to a conjugacy class of hyperbolic or loxodromic elements of the discrete isometry group $\Gamma$. The conjugacy classes can be directly computed from generators which define the Dirichlet fundamental domain of the $\mathrm{CH}$ manifold

Let $g_{i},(i=1, \ldots, N)$ be the generators and $\mathcal{I}$ be the identity. In general these generators are not independent. They obey a set of relations

\footnotetext{
${ }^{1}$ Given a set of generators $a$ and $b$ for the fundamental group of a torus, a closed curve which connects a point $x$ in the torus with $a x$ is called a meridian and another curve which connects a point $x$ with $b x$ is called a longitude.

${ }^{2}$ If $C$ connects a point $x$ with another point $(p a+q b) x$ where $p$ and $q$ are co-prime integer, $C$ is called a $(p, q)$ curve.
} 

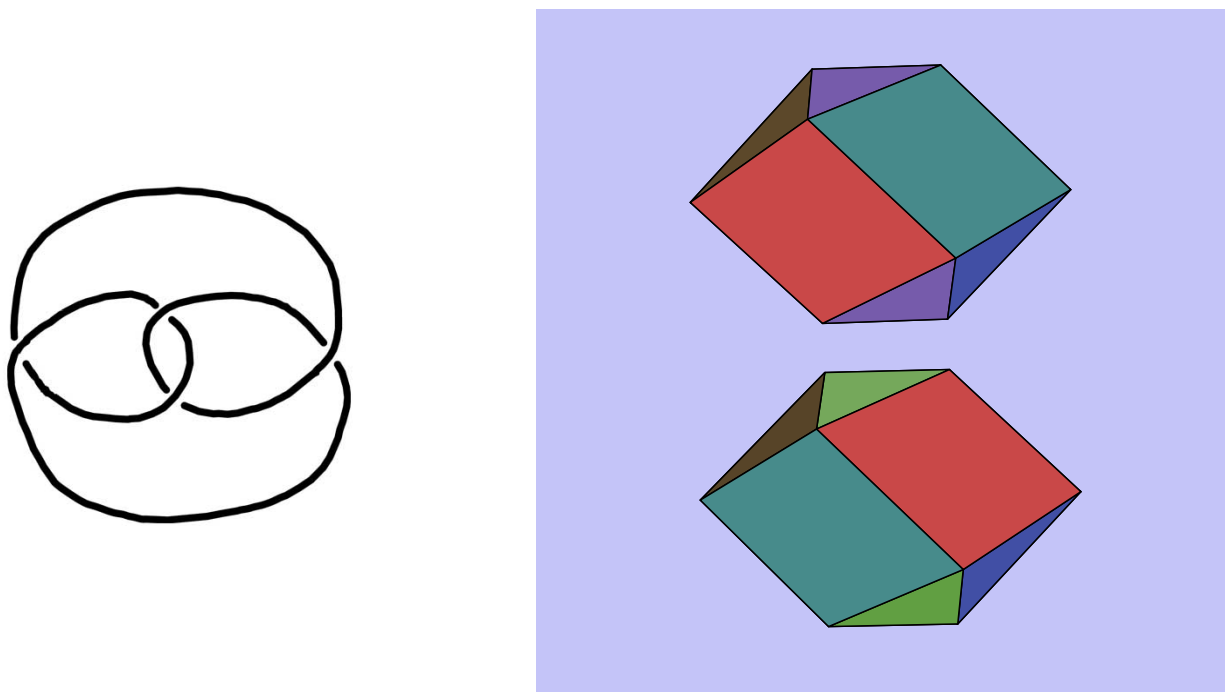

FIG. 2. "Figure eight knot" (left) and the Dirichlet domain of a cusped manifold m004 viewed from two opposite directions in the Klein(projective) coordinates where geodesics and planes are mapped into their Euclidean counterparts(right). The two vertices on the left and right edges of the polyhedron which are identified by an element of the discrete isometry group correspond to a cusped point. Colors on the faces correspond to the identification maps. One obtaines the Dirichlet domain of m003 by interchanging colors on quadrilateral faces in the lower(or upper) right figure.

$$
\prod g_{i_{1}} g_{i_{2}} \ldots g_{i_{n}}=\mathcal{I}
$$

which describe the fundamental group of $M$. Since all the elements of $\Gamma$ can be represented by certain products of generators, an element $g \in \Gamma$ can be written

$$
g=g_{i_{1}} g_{i_{2}} \ldots g_{i_{n}},
$$

which may be called a "word" . Using relations, each word can be shorten to a word with minimum length. Furthermore, all cyclic permutations of a product of generators belonging to the same conjugacy class can be eliminated. Thus conjugacy classes of $\Gamma$ can be computed by generating words with lowest possible length to some threshold length which are reduced by using either relations (7) among the generators or cyclic permutations of the product.

In practice, we introduce a cutoff length $l_{c u t}$ depending on the CPU power because the number of periodic orbits grows exponentially in $l$ which is a direct consequence of the exponential proliferation of tiles (copies of the fundamental domain) in tessellation. Although it is natural to expect a long length for a conjugacy class described in a word with many letters, there is no guarantee that all the the periodic orbits with length less than $l_{c u t}$ is actually computed or not for a certain threshold of length of the word.

Suppose that each word as a transformation that acts on the Dirichlet fundamental domain $D$. For example, $D$ is transformed to $D^{\prime}=g D$ by an element $g$. We can consider $g D^{\prime}$ s as tessellating tiles in the universal covering space. If the geodesic distance $d$ between the center (basepoint) of $D$ and that of $g D$ is large, we can expect a long periodic orbit that corresponds to the conjugacy class of $g$. Tessellating tiles to sufficiently long distance $d>l_{\text {cut }}$ makes it possible to compute the complex primitive length spectra $\left\{L_{j}=l_{j} \exp \left(i \phi_{j}\right), \mid l_{j}<l_{c u t}\right\}$ where $l_{j}$ is the real length of the periodic orbit of a conjugacy class with one winding number and $\phi_{j}$ is the phase of the corresponding transformation. We also compute multiplicity number $m\left(l_{j}\right)$ which counts the number of orbits having the same $l_{j}$ and $\phi_{j}$.

In general, the lower limit of the distance $d$ for computing a complete set of length spectrum for a fixed $l_{\text {cut }}$ is not known but the following fact has been proved by Hodgson and Weeks [21]. In order to compute a length spectra of a $\mathrm{CH} 3$-manifold (or 3-orbifold) with length less than $l$, it suffices to compute elements $\{g\}$ satisfying

$$
d(x, g x)<2 \cosh ^{-1}(\cosh R \cosh l / 2),
$$



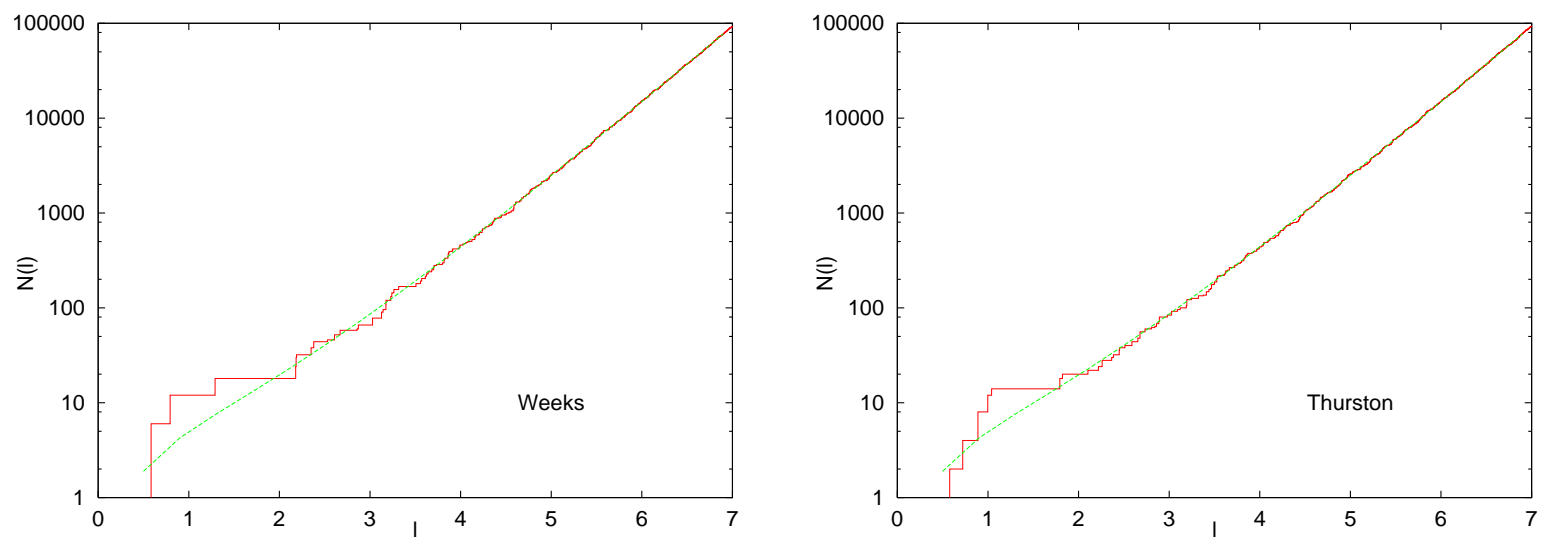

FIG. 3. The classical staircases $N(l)$ for the Weeks manifold and the Thurston manifold with the asymptotic distribution (10).

where $x$ is a basepoint and $R$ is the spine radius? 3 . Note that there is a unique geodesic which lies on an invariant axis for each hyperbolic or loxodromic element. SnapPea can compute length spectra of CH spaces either by the "rigorous method" based on the inequality (9) or the "quick and dirty" method by setting the tessellating radius $d$ by hand. I have used the former method for manifolds with small volume $(<1.42)$, but the latter method $\left(d=l_{\text {cut }}+0.5\right)$ has been also used for some manifolds with large volume $(>1.42)$ since the tessellating radius given by the former method is sometimes so large that the computation time becomes too long. The detailed algorithm is summarized in appendix A.

The asymptotic behavior of the classical staircase $N(l)$ which counts the number of primitive periodic orbits with length equal to or less than $l$ for $\mathrm{CH}$ 3-spaces can be written in terms of $l$ and the topological entropy $\tau$ [22]

$$
N(l) \sim \operatorname{Ei}(\tau l) \sim \frac{\exp (\tau l)}{\tau l}, \quad l \rightarrow \infty
$$

The topological entropy for $D$-dimensional $\mathrm{CH}$ spaces is given by $\tau=D-1$. A larger topological entropy implies that the efficiency in computation of periodic orbit is much less for higher dimensional cases [10].

In figure 3, the computed classical staircases $\left(l_{c u t}=7.0\right)$ are compared with the asymptotic formula for the smallest (Weeks) manifold and the second smallest (Thurston) manifold. For both cases, an asymptotic behavior is already observed at $l \sim 3.5$.

Although the asymptotic behavior of the classical staircase $N(l)$ does not depend on the topology of the manifold, the multiplicity number $m(l)$ does. In fact, it was Aurich and Steiner who firstly noticed that the locally averaged multiplicity number

$$
<m(l)>=\frac{1}{N} \sum_{l-\Delta l / 2<l_{i}<l+\Delta l / 2} g\left(l_{i}\right), \quad(N=\text { total number of terms })
$$

grows exponentially $<m(l)>\sim e^{l / 2} / l$ as $l \rightarrow \infty$ for arithmetic 2-spaces (manifolds and orbifolds) 23,7,24, since the length $l$ of the periodic orbits are determined by algebraic integers in the form $2 \cosh (l / 2)=$ algebraic integer 1 . The failure of application of the random matrix theory to some $\mathrm{CH}$ spaces may be attributed to the arithmetic property. For non-arithmetic spaces, one expects that the multiplicities are determined by the symmetries (elements of the isometry group) of the space. However, in the case of a non-arithmetic 3 -orbifold, it has been found that $<m(l)>$

\footnotetext{
${ }^{3}$ Spine radius $R$ is equal to the maximum over all the Dirichlet fundamental domain's edges of the minimum distance from the edge to the basepoint. Note that $\mathrm{R}$ is finite even for a cusped co-finite manifold.

${ }^{4}$ For a two-dimensional space, the classical staircase has an asymptotic form $N(l) \sim \exp (l) / l$. On the other hand, the classical staircase for distinct periodic orbits has a form $\hat{N}(l) \sim \exp (l / 2)$ for arithmetic systems. Because $m(l) d \hat{N}=d N$ we have $m(l) \sim \exp (l / 2) / l$ as $l \rightarrow \infty$.
} 


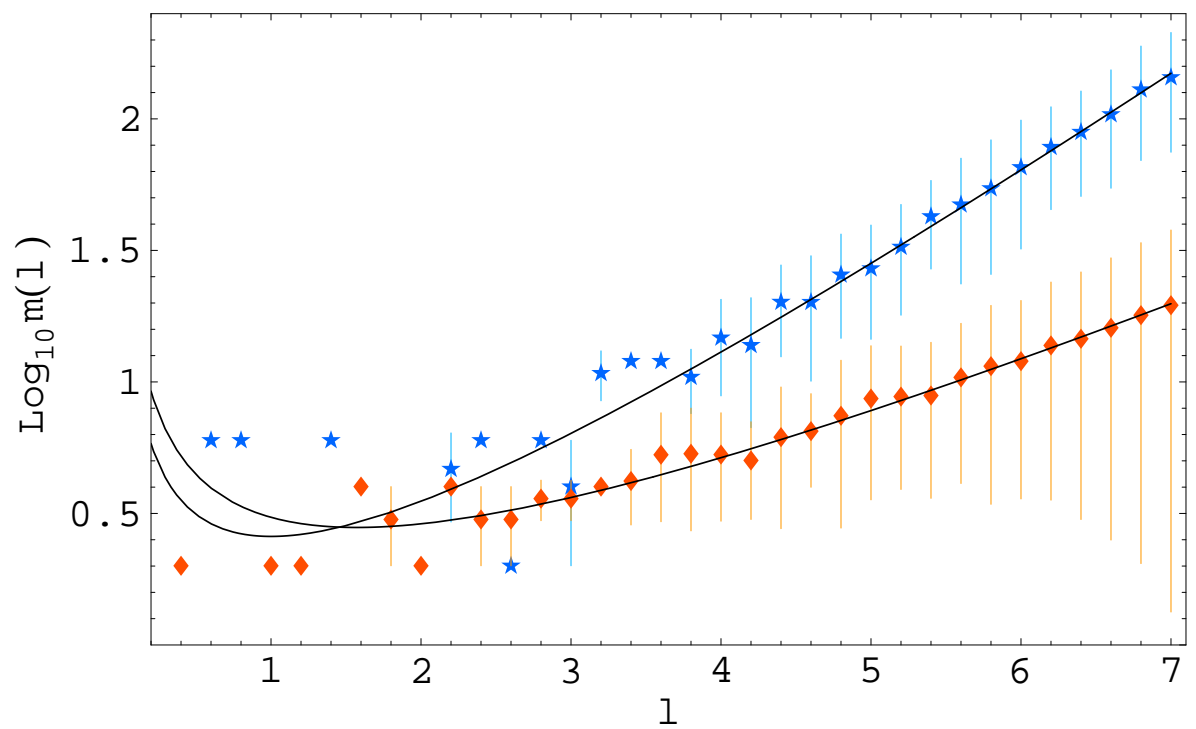

FIG. 4. Plots of locally averaged multiplicities $(\Delta l=0.2)$ with one-sigma errors and the fitting curves for the Weeks manifold m003(-3,1) which is arithmetic (star) and a non-arithmetic manifold m004(1,2) (diamond). The fitting curves $(a \exp (l) / l$ for the former and $\exp (b l) /(c l)$ for the latter $)$ are obtained by the least square method using data $3.0<l<7.0$.

grows exponentially in the form $e^{b l} /(c l)$ where $b$ and $c$ are fitting parameters [10]. This fact might implies that the symmetries of long periodic orbits are much larger than that of the space even in the case of non-arithmetic systems.

For 3-manifolds, one can expect that the property of $\langle m(l)\rangle$ for 3-orbifold also holds. The locally averaged multiplicities $(\Delta l=0.2)$ for the smallest twelve examples which include seven arithmetic and five non-arithmetic 3 -manifolds [25] have been numerically computed using SnapPea. From figure 4 , one can see that the difference in the behavior of $\langle m(l)\rangle$ between the non-arithmetic manifold and the arithmetic one is manifest. As observed in 3-orbifolds, averaged multiplicities behave as

$$
\begin{array}{rlrl}
<m(l)> & =a \frac{\exp l}{l}, \quad & \text { (arithmetic) } \\
& =\frac{\exp b l}{c l}, \quad \text { (non-arithmetic) }
\end{array}
$$

where $a$ depends on the discrete isometry group while $b$ and $c$ are fitting parameters. From table I, one observes that arithmetic manifolds having a larger symmetry group have a larger value of $a$. The growth rates for non-arithmetic manifolds $(b \sim 0.56)$ are always less than that for arithmetic manifolds $(b=1)$ but nevertheless exponential.

\section{PERIODIC ORBIT SUM METHOD AND SPECTRAL STAIRCASE}

Gutzwiller's periodic orbit theory provides a semiclassical quantization rule for classically chaotic systems. The theory is expressed in form of a semiclassical approximation $(\hbar \rightarrow 0)$ of the trace of the energy Green's operator (resolvent operator) $\hat{G_{E}}=(\Delta+E)^{-1}$ in terms of the length of periodic orbits (geodesics) $\left\{L_{i}\right\}$ which is known as the Gutzwiller trace formula [11]. For the dynamical system of a free massive particle on a $\mathrm{CH}$ space known as the Hadamard-Gutzwiller model, the periodic orbits give the exact eigenvalues, and the relation is no longer semi-classical approximation. In mathematical literature, the trace formula is known as the Selberg trace formula [12]. In what follows we consider only orientable $\mathrm{CH}$ 3-manifolds(denoted as $\mathrm{CH}$ manifolds) (for general cases including orbifolds, see 10$]$ ). The Selberg trace formula for a $\mathrm{CH}$ manifold $M=\mathbb{H}^{3} / \Gamma$ ( $\Gamma$ is a discrete isometry group containing only hyperbolic or loxodromic elements) can be written as

$$
\operatorname{Tr}\left(\hat{G}_{E}-\hat{G}_{E^{\prime}}\right)=-\frac{v(M)}{4 \pi i}\left(p-p^{\prime}\right)
$$




\begin{tabular}{|c|c|c|c|c|c|c|}
\hline manifold & volume & $\mathrm{A} / \mathrm{N}$ & G & $a$ & $b$ & $c$ \\
\hline $\mathrm{m} 003(-3,1)$ & 0.9427 & $\mathrm{~A}$ & D6 & 0.9514 & - & - \\
\hline m003(-2,3) & 0.9814 & $\bar{A}$ & D2 & 0.5667 & - & - \\
\hline $\mathrm{m} 007(3,1)$ & 1.0149 & $\mathrm{~A}$ & $\mathrm{D} 2 \dagger$ & 0.7108 & - & $\overline{-}$ \\
\hline m003(-4,3) & 1.2637 & $\mathrm{~A}$ & $\mathrm{D} 4$ & 0.8364 & - & - \\
\hline $\mathrm{m} 004(6,1)$ & 1.2845 & $\bar{A}$ & D2 & 0.6066 & - & - \\
\hline $\mathrm{m} 004(1,2)$ & 1.3985 & $\mathrm{~N}$ & D2 & - & 0.6360 & 0.6180 \\
\hline $\begin{array}{l}\mathrm{m} 009(4,1) \\
\end{array}$ & 1.4141 & $\mathrm{~A}$ & D2 & 0.5362 & - & - \\
\hline $\mathrm{m} 003(-3,4)$ & 1.4141 & $\mathrm{~A}$ & D2 & 0.5655 & - & \\
\hline m003(-4,1) & 1.4236 & $\mathrm{~N}$ & D2 & - & 0.5933 & 0.5912 \\
\hline $\mathrm{m} 003(3,2)$ & 1.4407 & $\mathrm{~N}$ & D2 & - & 0.6018 & 0.5532 \\
\hline $\begin{array}{l}\mathrm{m} 004(7,1) \\
\end{array}$ & 1.4638 & $\mathrm{~N}$ & D2 & - & 0.5693 & 0.4829 \\
\hline $\mathrm{m} 004(5,2)$ & 1.5295 & $\mathrm{~N}$ & D2 & - & 0.5780 & 0.5590 \\
\hline
\end{tabular}

TABLE I. Coefficients of the fitting curves which describe the average behavior of locally averaged multiplicities $<m(l)>$ for arithmetic (A) and non-arithmetic (N) 3-manifolds. G denotes the isometry group (symmetry group). Fitting parameters $a, b$ and $c$ are obtained by the least square method using data $3.0<l<7.0$. $\dagger$ For m007(3,1), the isometry group may be larger than D2.

$$
-\sum_{\left\{g_{\tau}\right\}} \frac{l\left(g_{\tau_{0}}\right)}{4\left(\cosh l\left(g_{\tau}\right)-\cos \phi\left(g_{\tau}\right)\right)}\left(\frac{\exp \left(-i p l\left(g_{\tau}\right)\right)}{i p}-\frac{\exp \left(-i p^{\prime} l\left(g_{\tau}\right)\right)}{i p^{\prime}}\right)
$$

where $p^{2}=E-1, v(M)$ denotes the volume of $M, l\left(g_{\tau}\right)$ is the (real) length of the periodic orbit of transformation $g_{\tau} \in \Gamma . g_{\tau_{0}}$ is a transformation that gives the shortest length of the periodic orbit $l\left(g_{\tau_{0}}\right)$ which commutes with $g_{\tau}$. $\phi\left(g_{\tau}\right)$ is the phase of the transformation $g_{\tau}$. The sum in (14) extends over $\Gamma$ conjugacy classes

$$
\left\{g_{\tau}\right\}:=\left\{g_{\tau}^{\prime} \mid g_{\tau}^{\prime}=h g_{\tau} h^{-1}, h \in \Gamma\right\}
$$

of hyperbolic $(\phi=0)$ or loxodromic elements $(\phi \neq 0)$. However the periodic orbit sum in (14) which is known as Maaß-Selberg series converges at only complex energy such that $\operatorname{Im} p<-1$ and $\operatorname{Im} p^{\prime}<-1$. In order to obtain real eigenvalues, one needs to multiply the trace by some suitable analytic "smoothing" function $h(q)$ that satisfies:

(i): $h(q)=h(-q)$;

(ii) $h(q)=\mathcal{O}\left(|q|^{-3-\delta}\right)$ for $\delta>0$ as $|q| \rightarrow \infty$;

(iii): $h(q)$ is analytic in the strip $|\operatorname{Im} q|<1+\epsilon$ for $\epsilon>0$.

Multiplying (14) by $q h(q) /(\pi i)$ and integrating it over $q$ from $-\infty$ to $\infty$, one obtains the general Selberg trace formula,

$$
\begin{aligned}
\sum_{n=0}^{\infty} h\left(p_{n}\right)= & -\frac{v(M)}{2 \pi} \tilde{h}^{\prime \prime}(0) \\
& +\sum_{\left\{g_{\tau}\right\}} \frac{l\left(g_{\tau_{0}}\right)}{2\left(\cosh l\left(g_{\tau}\right)-\cos \phi\left(g_{\tau}\right)\right)} \tilde{h}\left(l\left(g_{\tau}\right)\right)
\end{aligned}
$$

where

$$
\tilde{h}(l)=\frac{1}{2 \pi} \int_{\infty}^{\infty} d q h(q) \exp (-i q l)
$$

and $\tilde{h}^{\prime \prime}(0)$ is the second derivative of $\tilde{h}$ and $p_{n}$ denotes a wavenumber of the corresponding eigenmode. The sum in (16) is absolutely convergent for any real eigenvalues $E_{n}=p_{n}^{2}+1$. One can obtain various functions of eigenvalues such as heat kernels and energy level densities from periodic orbits by choosing an appropriate "smoothing" function $h(q)$.

In order to obtain eigenvalues, a simple approach is to compute the spectral staircase

$$
N(E)=\sum_{n=0}^{\infty} \theta\left(E-E_{n}\right)
$$


where $E_{0}=0, E_{1}, E_{2}, \cdots$ are the eigenvalues of the Laplacian and $\theta$ is the Heaviside function [4]. To explore supercurvature modes $u_{E}, 0 \leq E<1$, we choose the "smoothing" function of $N(E)$ as

$$
\begin{aligned}
h_{p, \epsilon}\left(p^{\prime}\right) & =\frac{1}{2}\left(1-\operatorname{Erf}\left(\frac{E^{\prime}-E}{\epsilon^{2}}\right)\right), \\
& =\frac{1}{2}\left(1-\operatorname{Erf}\left(\frac{p^{2}-p^{2}}{\epsilon^{2}}\right)\right),
\end{aligned}
$$

which is real for $E>0$. Note that $h_{p}\left(p^{\prime}\right)$ satisfies all the conditions (i) to (iii). By taking the limit $\epsilon \rightarrow 0$, one obtains the spectral staircase

$$
N(E)=\lim _{\epsilon \rightarrow 0} \sum_{n=0}^{\infty} h_{p, \epsilon}\left(p_{n}\right)
$$

Let us first estimate the behavior of the trace in (16). From a straightforward calculation, the zero-length contribution can be written as

$$
\begin{aligned}
\tilde{h}_{p, \epsilon}^{\prime \prime}(0)= & -\frac{\epsilon^{3}}{6 \pi^{\frac{3}{2}}} \exp \left(-p^{4} / \epsilon^{4}\right) \\
& \times\left(\frac{\sqrt{2} \pi}{4}(\Gamma(3 / 4))^{-1} F\left(5 / 4,1 / 2, p^{4} / \epsilon^{4}\right)+\frac{3 p^{2}}{2 \epsilon^{2}} \Gamma(3 / 4) F\left(7 / 4,3 / 2, p^{4} / \epsilon^{4}\right)\right),
\end{aligned}
$$

where $\Gamma(x)$ is the Gamma function and $F(a, b, z)$ is the confluent hypergeometric function. For $x=p^{4} / \epsilon^{4} \rightarrow \infty$, it is asymptotically expanded as

$$
\begin{aligned}
F(a, b, x) & =\frac{\Gamma(b)}{\Gamma(b-a)} e^{i \pi a} x^{-a}\left\{\sum_{n=0}^{R-1} \frac{(a)_{n}(1+a-b)_{n}}{n !}(-x)^{-n}+\mathcal{O}\left(x^{-R}\right)\right\} \\
& +\frac{\Gamma(b)}{\Gamma(a)} e^{x} x^{a-b}\left\{\sum_{n=0}^{S-1} \frac{(b-a)_{n}(1-a)_{n}}{n !}(x)^{-n}+\mathcal{O}\left(x^{-S}\right)\right\},
\end{aligned}
$$

where $(a)_{m} \equiv \Gamma(a+m) / \Gamma(a)$. From (21) and (22), in the lowest order, we have the average part,

$$
\hat{N}(p)=\lim _{\epsilon \rightarrow 0}-\frac{v(M)}{2 \pi} \tilde{h}_{p, \epsilon}^{\prime \prime}(0)=\frac{v(M)}{6 \pi^{2}}\left|p^{2}\right|^{\frac{3}{2}},
$$

that gives the dominant term in the Weyl asymptotic formula for $p>>1$.

Next, we estimate the oscillating term in (16)

$$
\tilde{h}_{p, \epsilon}(l)=\alpha \int_{-\infty}^{\infty} d q \exp (-i q l) q \exp \left(-\frac{\left(q^{2}-p^{2}\right)^{2}}{\epsilon^{4}}\right), \quad \alpha \equiv \frac{i}{l \epsilon^{2} \pi^{3 / 2}} .
$$

In the long orbit-length limit $l \gg 1$ with $p>0$, the integrand in Eq.24 oscillates so rapidly that the dominant contribution comes from $q \sim p$, or $-p$ where $\left(q^{2}-p^{2}\right)^{2} / \epsilon^{4}$ can be approximately given as $-4(q-p)^{2} p^{2} / \epsilon^{4}$. Then (24) can be written as

$$
\tilde{h}_{p, \epsilon}(l) \sim \frac{1}{\pi l} \sin (p l) \exp \left(\frac{-\epsilon^{4} l^{2}}{16 p^{2}}\right) .
$$

Thus each periodic orbit corresponds to a wave with wavelength $2 \pi / l$ and an amplitude which is exponentially suppressed with an increase in $l$ or a decrease in $p$. For a finite subset of length spectra $l_{j}<l_{c u t}$, the appropriate choice for the smoothing scale is given by $\epsilon=\alpha p^{1 / 2}$ for $p^{2}>0$ where $\alpha$ depends on $l_{\text {cut }}$ since for a reasonable value of the proportional factor $\alpha$ all the contributions from periodic orbits with large length $l>l_{\text {cut }}$ can be negligible. For $p^{2}<0$, an optimal choice can be obtained from a numerical computation of Eq.(24) directly. Comparing the obtained smoothed spectral staircase with the one based on the computed "true" eigenvalues using the direct boundary element method (DBEM), it is numerically found that for $l_{c u t}=7.0$, an appropriate smoothing scale is given by 

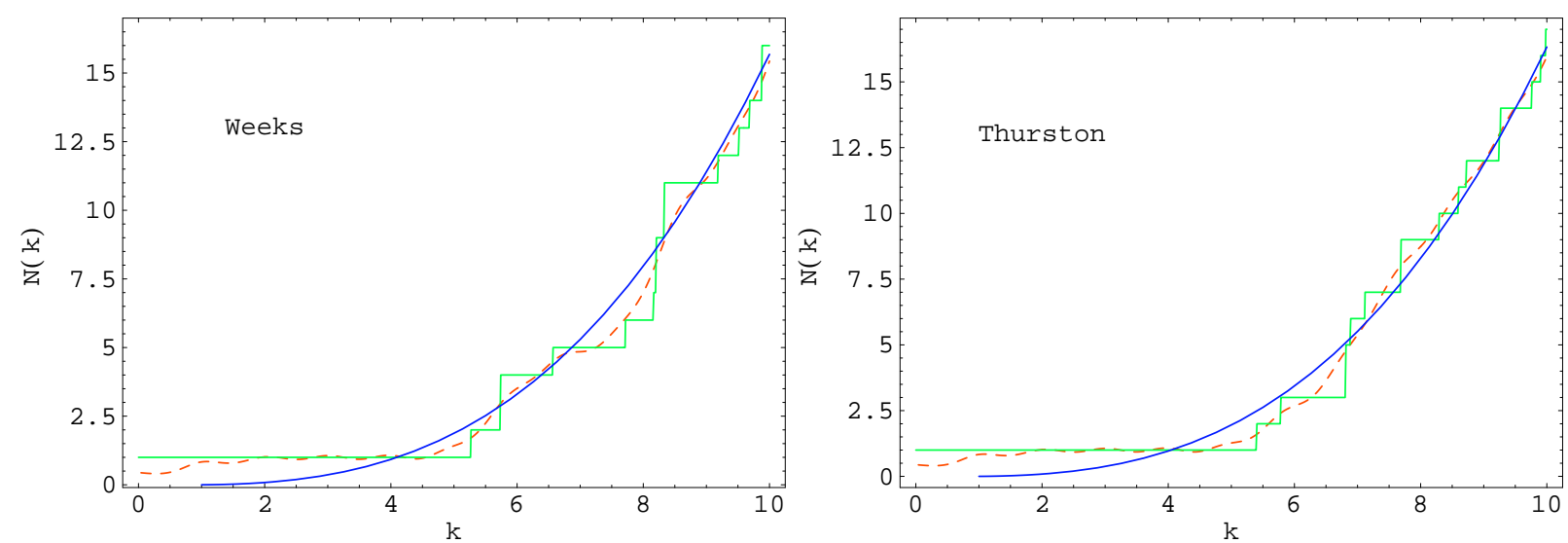

FIG. 5. The spectral staircases $N(k)$ for the Weeks manifold and the Thurston manifold obtained by the DBEM are compared with the average parts $\hat{N}(k)$ (solid curves), namely, Weyl's asymptotic formula (23) and smoothed spectral staircase (dotted curves) obtained by the periodic orbit sum method(POSM) using all periodic orbits $l<7.0$.

\begin{tabular}{|c|c|c|c|c|c|}
\hline manifold & volume & $k_{1}(\mathrm{DBEM})$ & $k_{1}(\mathrm{POSM})$ & $\mathrm{m}\left(k_{1}\right)$ & $\Delta k_{1} / k_{1}$ \\
\hline $\mathrm{m} 003(-3,1)$ & 0.9427 & 5.27 & 5.10 & 1 & 0.03 \\
\hline $\mathrm{m} 003(-2,3)$ & 0.9814 & 5.40 & 5.34 & 1 & 0.01 \\
\hline $\mathrm{m} 007(3,1)$ & 1.0149 & 5.29 & 5.37 & 1 & 0.02 \\
\hline $\mathrm{m} 003(-4,3)$ & 1.2637 & 4.58 & 4.31 & 2 & 0.06 \\
\hline $\mathrm{m} 004(6,1)$ & 1.2845 & 4.53 & 4.35 & 1 & 0.04 \\
\hline $\mathrm{m} 004(1,2)$ & 1.3985 & 4.03 & 3.93 & 1 & 0.02 \\
\hline m009(4,1) & 1.4141 & 5.26 & 4.84 & 2 & 0.08 \\
\hline
\end{tabular}

TABLE II. The first (non-zero) wavenumbers $k_{1}=E_{1}^{1 / 2}$ are calculated using the DBEM and using the POSM for seven smallest manifolds. $k_{1}$ 's agree with relative accuracy $\Delta k_{1} / k_{1}=0.01-0.08$. The multiplicity number of the first eigenmode is calculated using the DBEM.

$$
\epsilon(k)=\left\{\begin{array}{l}
0.116 k^{2}+0.184 k+1.2 \quad(k<1) \\
0.832 k^{1 / 2}+0.668 \quad(k \geq 1)
\end{array},\right.
$$

where $E=k^{2}=p^{2}+1$. The eigenvalues can be computed by searching $E$ at which $N(E)-0.5$ becomes positive integer. It should be emphasised that the precision of computation depends on the value of $l_{c u t}$ which determines the resolution scale in the eigenvalue spectra.

In order to get eigenvalues from the smoothed spectral staircase, one must take into account the effect of the multiplicity number(degeneracy number) for each eigenvalue since the spectral staircase is smoothed on larger scales for degenerated modes. Therefore, the numerical accuracy becomes worse if the eigenmode has a large multiplicity number. Fortunately, the order of the symmetry group is not so large for a small manifold (volume $<3$ ). For instance, of the twelve smallest examples, nine manifolds have a symmetry group with order 4 . If one assumes that the multiplicity number is either 1 or 2 then the deviation $\Delta k$ from a precise value is approximately given by

$$
\frac{1}{2}\left(1-\operatorname{Erf}\left(\frac{k^{2}-(k+\Delta k)^{2}}{\epsilon^{2}}\right)\right)=\frac{1 \pm 0.5}{2} .
$$

For instance, if one uses a length spectrum $l<7.0$ then (27) gives $\Delta k=0.30,0.33$ for $k=5.0,3.0$, respectively. If one permits the multiplicity number as much as 6 , then the expected precision becomes $\Delta k=0.49,0.54$ for $k=5.0,3.0$, respectively.

We can see from table II that the first eigenvalues calculated by using length spectra $l<7.0$ for some smallest known $\mathrm{CH}$ manifolds lie within several per-cent of those obtained by the DBEM. Note that the eigenvalues are also consistent with those obtained by the Trefftz method [17]. For two examples in which the first non-zero mode is degenerated, 
the eigenvalues are much shifted to lower values owing to the smoothing effect. From figure 5 , one can see that the curves of the obtained smoothed spectral stairs cross the "true" stairs at almost half height. A slight deviation from the average part of the spectral staircase is caused by the interference of waves each one of which corresponds to a periodic orbit.

\section{FIRST EIGENVALUE AND GEOMETRICAL QUANTITIES}

The estimate of the first (non-zero) eigenvalue $E_{1}=k_{1}^{2}$ of the Laplace-Beltrami operator plays a critical role in describing the global topology and geometry of manifolds. A number of estimates of $E_{1}$ for $n$-dimensional compact Riemannian manifolds $M$ using diffeomorphism-invariant quantities have been proved in mathematical literature.

First of all, we consider the relation between the first eigenvalue $E_{1}$ and the diameter $d$ which is defined as the maximum of the minimum geodesic distance between two arbitrary points on $M$. Various analytic upper and lower bounds of $E_{1}$ in terms of $d$ have been known. Suppose $M$ with Ricci curvature bounded below by $-L(L>0)$. Cheng and Zhou proved that $E_{1}$ satisfies

$$
E_{1} \geq \max \left[\frac{1}{2} \frac{\pi^{2}}{d^{2}}-\frac{1}{4} L, \sqrt{\frac{\pi^{4}}{d^{2}}+\frac{L^{2}}{16}}-\frac{3}{4} L, \frac{\pi^{2}}{d^{2}} \exp \left(-C_{n} \sqrt{L d^{2}} / 2\right)\right],
$$

where $C_{n}=\max [\sqrt{n-1}, \sqrt{2}$ ] [26]. Another lower bound has been obtained by Lu [27]. Suppose that the Ricci curvature of $M$ is bounded below as $R_{a b} \geq-K g_{a b},(K \geq 0)$ for a some real number $K$ where $g_{a b}$ is the metric tensors of $M$. Then $E_{1}$ satisfies

$$
E_{1} \geq \max \left[\frac{\pi^{2}}{d^{2}}-K, \frac{8}{d^{2}}-\frac{K}{3}, \frac{8}{d^{2}} \exp \left(-\frac{d^{2} K}{8}\right), \frac{8}{d^{2}}\left(1+\frac{d}{3} \sqrt{K(n-1)}\right) \exp \left(-\frac{d}{2} \sqrt{K(n-1)}\right)\right] .
$$

As for upper bounds, the following theorem has been proved by Cheng [28]. Suppose M with Ricci curvature larger than $(n-1) c$, then we have

$$
E_{1} \leq \tilde{E}_{1}\left(V_{n}(c, d / 2)\right)
$$

where $V_{n}(c, r)$ denotes a geodesic ball with radius $r$ in the $n$-dimensional simply-connected space with sectional curvature $c$ and $\tilde{E}_{1}$ is the first Dirichlet eigenvalue. Setting $L=K=2, n=3$, and $c=-1$, we obtain the upper and lower bounds of $E_{1}$ for $\mathrm{CH} 3$-manifolds. For the upper bound, (30) gives a simple relation, $d \leq 2 \pi / \nu_{1}$, where $\nu_{1}^{2}=k_{1}^{2}-1$. The physical interpretation is clear: the wavelength $\lambda_{1} \equiv 2 \pi / \nu_{1}$ of the lowest non-zero mode must be larger than the diameter.

Now, we compare the first eigenvalues of 263 examples of $\mathrm{CH} 3$-manifolds with volume less than 3 which have the length of the shortest periodic orbit $l_{\min }>0.3$ (the Hodgson-Weeks census 21]) and of 45 other examples obtained by Dehn surgeries $(|p|<17,|q|<14)$ on a cusped manifold m0035 with the analytic bounds. The diameter of a $\mathrm{CH}$ 3 -manifold is given by the supremum of the outradius over all the basepoints, which has been numerically computed using the SnapPea kernel. The numerical accuracy is typically $\Delta d=0.03-0.09$ depending on the topology of the manifold.

As shown in figure 6, the eigenvalues are well described by an empirical fitting formula $\lambda_{1}=\beta d$, or

$$
k_{1}=\sqrt{1+\frac{4 \pi^{2}}{\beta^{2} d^{2}}}
$$

Applying the least square method for the 263 manifolds in the Hodgson-Weeks census (group A), and 53 manifolds obtained by Dehn surgeries on m003 (group B), the best-fit values $\beta=1.70,1.73$ have been obtained for each group,

\footnotetext{
${ }^{5}$ The Hodgson-Weeks census with volume less than 3 also includes 8 manifolds obtained by Dehn surgeries on m003.

${ }^{6}$ The outradius at a basepoint $x$ is equal to the minimum radius of the simply-connected ball which encloses the Dirichlet domain at $x$.

${ }^{7}$ I would like to thank J. Weeks for providing me a code to compute the diameter using the SnapPea kernel.
} 


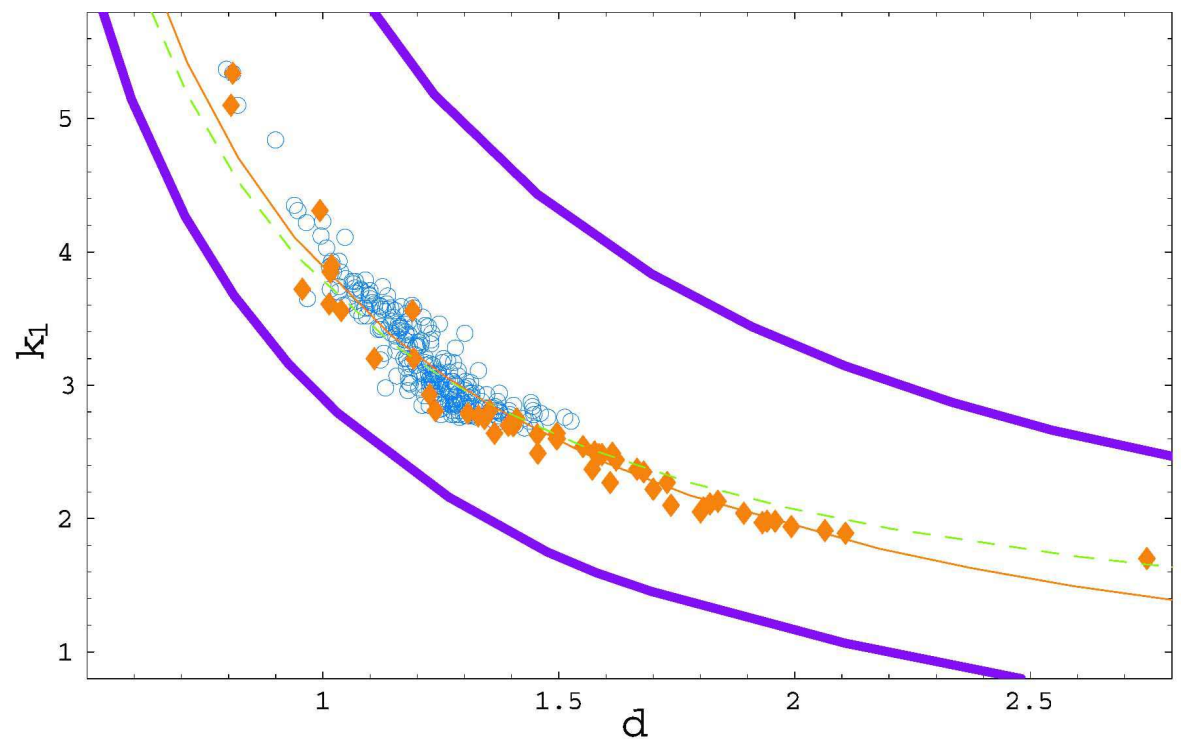

FIG. 6. Diameter $d$ versus $k_{1}$ for 263 examples of $\mathrm{CH} 3$-manifolds with $l_{\text {min }}>0.3$ and $v<3$ (group A, circle) and 52 examples that are obtained by performing Dehn surgeries (51 examples for $|p|,|q|<10$ plus 1 example $(p, q)=(16,13)$ group B, diamond) on a cusped manifold m003 with the best-fit curves for group B corresponding to (31) (dashed curve) assuming $k_{1}$ (cusp) $=1$ and (32) (solid curve) assuming $k_{1}$ (cusp) $=0.1$. The fitting curves also agree with the computed values for group A. The upper and lower thick curves denote the analytic bounds (28), (29) and (30).

respectively. Note that $\beta \sim 1.7$ is slightly larger than the values 1.3-1.6 for 12 examples in the previous result by Cornish and Spergel [17]. The deviation from the fitting formula (31) is found to be remarkably small (with one sigma error $\Delta k_{1}=0.18,0.19$ for each group, respectively), which implies the existence of much sharper bounds.

The empirical formula (31) asserts that no supercurvature modes $\left(\right.$ i.e. $k_{1}<1$ ) exist in the limit $d \rightarrow \infty$ where the manifold converges to the original cusped manifold. However, cusped manifolds may have some supercurvature modes even for those with small volume. Instead of (31), we consider a generalised empirical formula

$$
k_{1}=\sqrt{\left(k_{1}(\text { cusp })\right)^{2}+\frac{4 \pi^{2}}{\beta^{2} d^{2}}},
$$

where $k_{1}$ (cusp) $\leq 1$ is the smallest wavenumber for the original cusped manifold. Although no supercurvature modes were observed in this analysis, the non-existence of such modes in the limit $d \rightarrow \infty$ was not confirmed since the numerical accuracy becomes worse for manifolds with small $k_{1}$ and large $d$.

Next, we consider the relation between diameter $d$ and volume $v$ of the manifold. Since diameter is given by the supreme of the outradius (minimum radius of a sphere which circumscribes the Dirichlet domain) all over the basepoints, one expect that $v$ is estimated as the volume of a sphere (in a hyperbolic space) with radius $r$ somewhat smaller than $d$ if there is no region which resembles the neibourhood of a cusp ("thin part" ?) or equivalently $l_{\min }$ is sufficiently large. Suppose that $r=\alpha d$ with $\alpha<1$ then the volume of a sphere

$$
v=\pi(\sinh (2 \alpha d)-2 \alpha d)
$$

gives the approximate value of a CH manifold with diameter $d$. The best fit value for a sample of 79 manifolds with $l_{\text {min }}>0.5$ is $\alpha=0.69$. If a manifold has a "thin" part then the relation (33) is no longer valid since the diameter becomes too long. Let $v_{c}$ be the volume of a cusped manifold $M_{c}$ (with only one cusp) and $v(d)$ be the volume of a $\mathrm{CH}$ manifold $M$ obtained by a Dehn surgery on $M_{c}$. In the limit $d \rightarrow \infty$, one can show that the following approximation holds (see appendix B):

\footnotetext{
${ }^{8} \mathrm{~A}$ "thin" part is defined as a region where the injectivity radius(a half the minimum length of the periodic orbit) is short.
} 


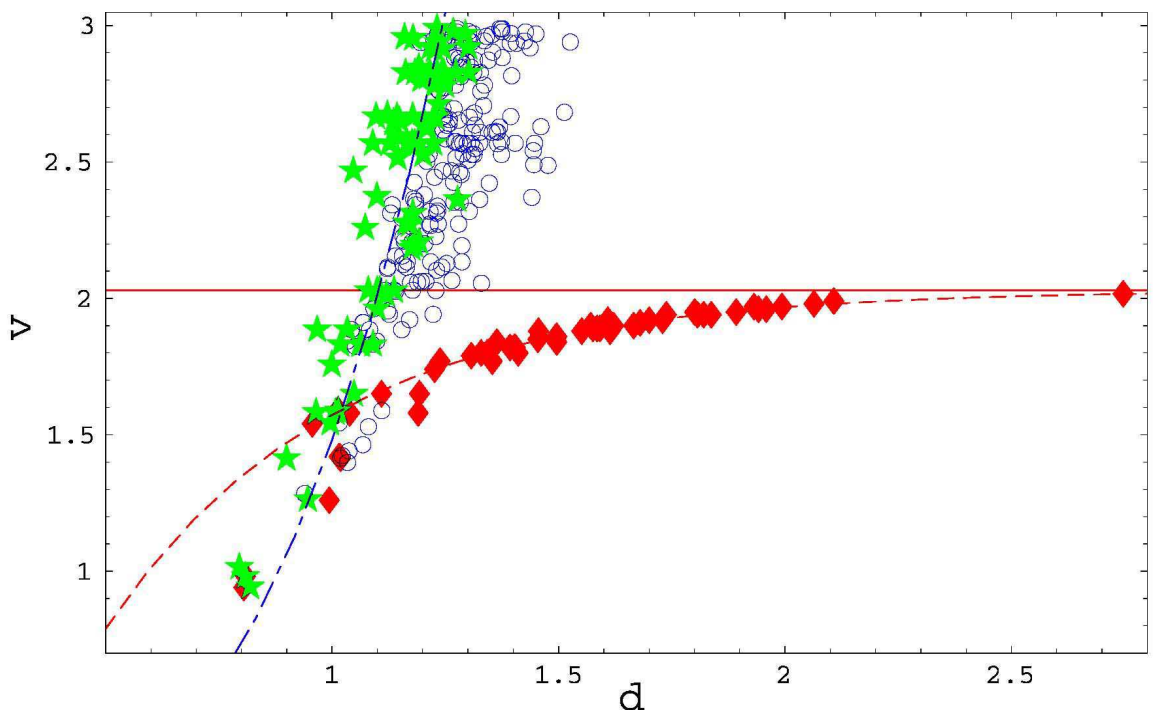

FIG. 7. Diameter $d$ versus volume $v$ for 79 manifolds $\left(l_{\min }>0.5\right)$ (star), 184 manifolds $\left(0.5>l_{\text {min }}>0.3\right)($ circle $)$ and 53 manifolds obtained by performing Dehn surgeries on m003 (group B, diamond) with the best-fit curves (33) (dashed-dotted curve) and (34) (dashed curve). The solid curve denotes the volume of m003.

$$
v(d)=v_{c}\left(1-\frac{\exp \left(-2\left(d-d_{0}\right)\right)}{\delta+1}\right)
$$

where $\delta$ denotes a ratio of the volume of the complementary part to that of the "thin" part and $d_{0}$ is the diameter of the complementary part. For a sample of 41 manifolds with diameter longer than 1.65 in group B, the best fits are $\delta=0.0$ and $d_{0}=0.25$. As shown in figure 7 , the volume-diameter relation for $\mathrm{CH}$ manifolds with large $l_{m i n}$ is well described by the fitting formula (33). As $l_{\text {min }}$ becomes smaller, or equivalently, $d$ becomes larger, a $\mathrm{CH}$ manifold $M$ converges to the original cusped manifold $M_{c}$ in which (34) holds.

Finally, we look into the relation between the first eigenvalue and the volume. For CH manifolds with sufficiently large $l_{\min },(32)$ and (33) give

$$
v\left(k_{1}\right)=\pi\left(\sinh \left(g\left(k_{1}\right)\right)-g\left(k_{1}\right)\right), \quad g\left(k_{1}\right)=\frac{4 \pi \alpha}{\beta \sqrt{\left(k_{1}\right)^{2}-\left(k_{1}(\operatorname{cusp})\right)^{2}}} .
$$

To be consistent with the Weyl's asymptotic formula which is valid for $k_{1}>>1$

$$
k_{1}(v)=\sqrt{\left(\frac{9 \pi^{2}}{v}\right)^{\frac{2}{3}}+1}
$$

the fitting parameters should satisfy $\alpha / \beta=3 \cdot 4^{-5 / 6} \pi^{-2 / 3} \approx 0.44$ which well agree with the numerically computed values $\alpha / \beta=0.69 /(1.6-1.7)=0.41-0.43$ provided that $k_{1}($ cusp $)=1$. One can see from figure 8 that both $(35)$ and (36) give a good estimate of the first eigenvalue $k_{1}^{2}$ for globally "slightly anisotropic" manifolds with $v<3$ and $l_{\text {min }}>0.5$. For manifolds with large $d, v$ and small $l_{\text {min }}, 35$ ) and (36) give incorrect estimates for $k_{1}$. In that case, the effect of the curvature cannot be negligible. In contrast to compact flat spaces, the volume of a sphere in hyperbolic space increases exponentially as the radius increases. Assuming that the relation (35) holds, for sufficiently globally "isotropic" $\mathrm{CH}$ manifolds (large $l_{\text {min }}$ ), $k_{1}$ is significantly larger than that for compact flat spaces with the same volume even if one assumes that $k_{1}($ cusp $) \sim 0$. However, for $\mathrm{CH}$ manifolds converging to the original cusped manifold $M_{c}$, the formula (35) has to be modified. If $l_{\text {min }}$ is sufficiently small and $d$ is large while keeping the volume finite then $M$ has a "thin" part similar to the neibourhood of a cusp. Then one can use an asymptotic formula (34) instead of (33).

$$
k_{1}(v)=\sqrt{\left(k_{1}(\operatorname{cusp})\right)^{2}+\frac{4 \pi^{2}}{\beta^{2}\left(d_{0}-\ln \left(1-v / v_{c}\right) / 2\right)^{2}}},
$$




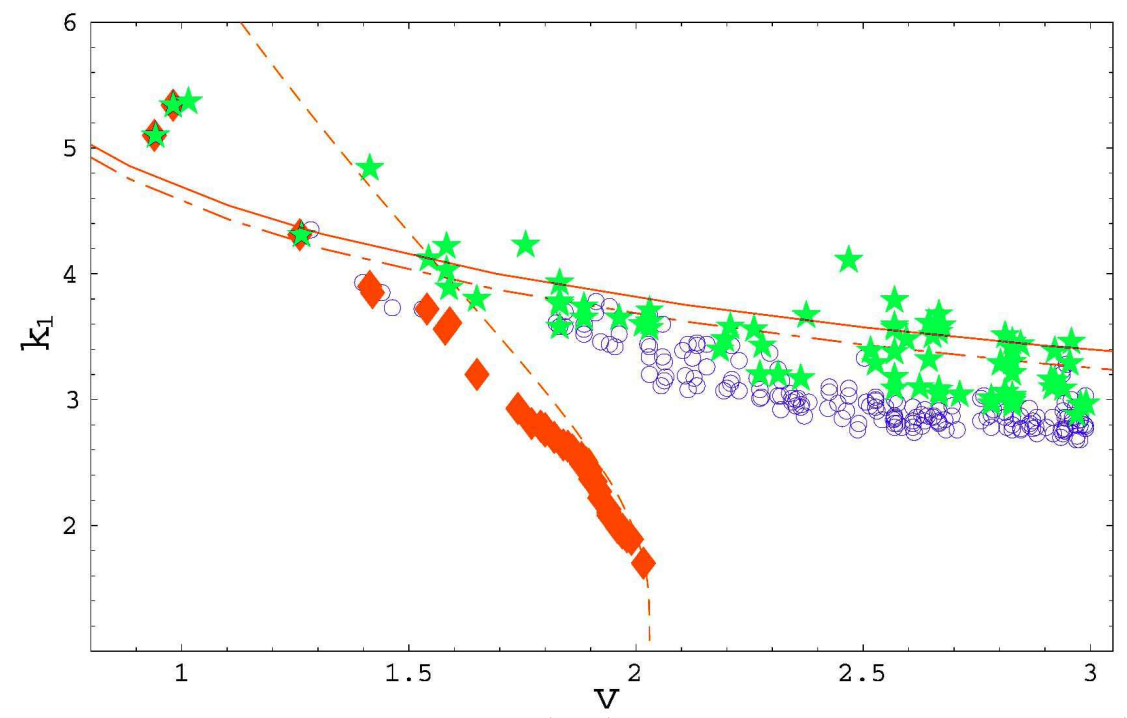

FIG. 8. Volume $v$ versus $k_{1}$ for 79 manifolds with $l_{\min }>0.5$ (star), 184 manifolds with $0.5>l_{\min }>0.3$ (circle) and 53 manifolds obtained by performing Dehn surgeries on m003 (group B, diamond) with fitting curves (35)(solid curve), (36)(dashed-dotted curve), and (37) (dashed curve) where $k_{1}$ (cusp) $=1$ is assumed.
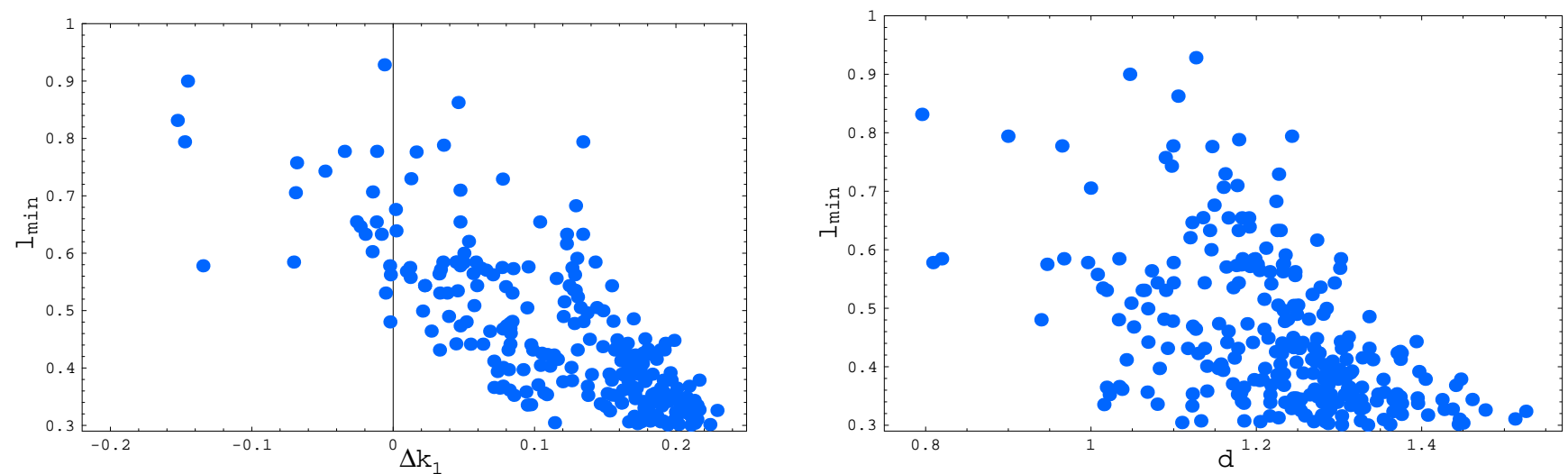

FIG. 9. The length of the shortest periodic orbit $l_{\text {min }}$ versus deviation $\Delta k_{1}$ from the fitting formula (35) where $k_{1}($ cusp) $=1$ is assumed(left) and $l_{\text {min }}$ versus diameter $d$ (right) for $263 \mathrm{CH}$ manifolds (group A). 
FIG. 10. Spectral staircases for m003(9,7) (dashed-dotted staircase, left) , m003(16,13) (solid staircase, right) and m003(16,13) without the shortest periodic orbit (dotted staircase, right) are shown in comparison with the corresponding Weyl's asymptotic formula (solid curves) which have been numerically computed by the POSM using all periodic orbits with length $l<7$. $N(k)=k$ perfectly fits the staircase of m003(16,13) for $k<5$ (thick line, right).

where $v_{c}$ is the volume of $M_{c}$. As shown in figure 9, $k_{1}$ shifts to a smaller value for manifolds with smaller $l_{\text {min }}$ which have larger $d$. In the limit $M \rightarrow M_{c}$, the length of periodic orbits of $M$ also converges to that of $M_{c}$ except for the shortest orbit whose length $l_{\text {min }}$ goes to zero. From the general Selberg trace formula (16), one can see that the wave which corresponds to the shortest orbit has a large amplitude $\sim 1 / \cosh l_{\min }$ with a long wavelength $\sim 2 \pi / l_{\text {min }}$. Therefore, the presence of a very short periodic orbit results in the deviation of the energy spectrum at low-energy region (small $k$ ) from the asymptotic distribution. To confirm this, the spectral staircase using the length spectra of m003(16,13) but the shortest periodic orbit is removed has been computed. Note that m003(16,13) is very similar to the original cusped manifold m003 having $d=2.75$ and $l_{\min }=0.0086$. As shown in figure 10, the computed spectrum staircase agrees well with Weyl's asymptotic formula. The computed spectrum may coincide with the one for a manifold in which the "thin" part is cut off. On the other hand, the spectral staircases for m003 $(9,7)$ and m003(16,13) which have small $l_{\text {min }}$ deviate from the average part (=Weyl's asymptotic formula(23)) (figure 10). These manifolds have a "thin" part which is virtually one-dimensional object. Let us remind that Weyl's asymptotic formula for a n-dimensional compact manifold $M$ is given by

$$
N(k) \sim \frac{\omega_{n} v(M) k^{n}}{(2 \pi)^{n}}, \quad k>>1,
$$

where $\omega_{n}=2 \pi^{n / 2} /(n \Gamma(n / 2))$ is the volume of the unit disk in the Euclidean n-space and $v(M)$ is the volume of $M$. If one assumes that the Weyl formula still holds for small $k$, then the spectrum in the low-energy region which corresponds to fluctuations on large scales for these manifolds can be approximately described by the Weyl formula for $n=1, N(k) \propto k / \pi$. For m003(16,13), it is numerically found that $N(k)=k$ provides a good fit for $k<5$. In the next section, we will measure the deviation from the asymptotic distribution using the low-lying eigenvalue spectra.

\section{SPECTRAL MEASUREMENT OF GLOBAL ANISOTROPY}

Among many possibilities, we should choose physically well-motivated quantities for measuring the global "anisotropy" in geometry in terms of eigenvalue spectra. First, we consider $\zeta$-function which is relevant to the cosmological microwave background anisotropy. The angular power spectra for $\mathrm{CH}$ universes are approximately written as [29]

$$
C_{l} \sim \sum_{i=1}^{\infty} \frac{F_{l}\left(k_{i}\right)}{k_{i}^{3}}
$$

where $F_{l}(k)$ can be approximated as a polynomial function of $k$. In order to measure the "anisotropy", we will define the following parameter,

$$
\Delta(s) \equiv \zeta(s) / \zeta_{w}(s)=\sum_{i=1}^{\infty} k_{i}^{-2 s} / \sum_{i=1}^{\infty} k_{w i}^{-2 s},
$$

where $k_{i}^{2}$ are the eigenvalues of the Laplace-Beltrami operator on a CH 3-manifold $M$ with volume $v$ and $k_{w i}^{2}$ are the eigenvalues obeying Weyl's asymptotic formula with volume $v$ (36). Note that the zero-mode $k_{0}=0$ is not included in the summation. Here we only consider the case $s>1$ which ensures the convergence of the sum. The numerical result shows the clear difference between the "slightly anisotropic" manifold m003(-3,1) $\left(d=0.82, l_{\min }=0.58\right)$ , "somewhat anisotropic" manifold m003 $(9,7)\left(d=2.11, l_{\text {min }}=0.028\right)$ and "very anisotropic" manifold m003(16,13) $\left(d=2.75, l_{\min }=0.0086\right)$ (figure 11). The presence of the fluctuations on large scales in these "anisotropic" manifolds shifts the corresponding $\zeta$-function to a larger value. For the case in which the shortest periodic orbit is removed from the length spectra of m003(16,13), one can see that the spectrum coincides with that obeying Weyl's asymptotic formula. 

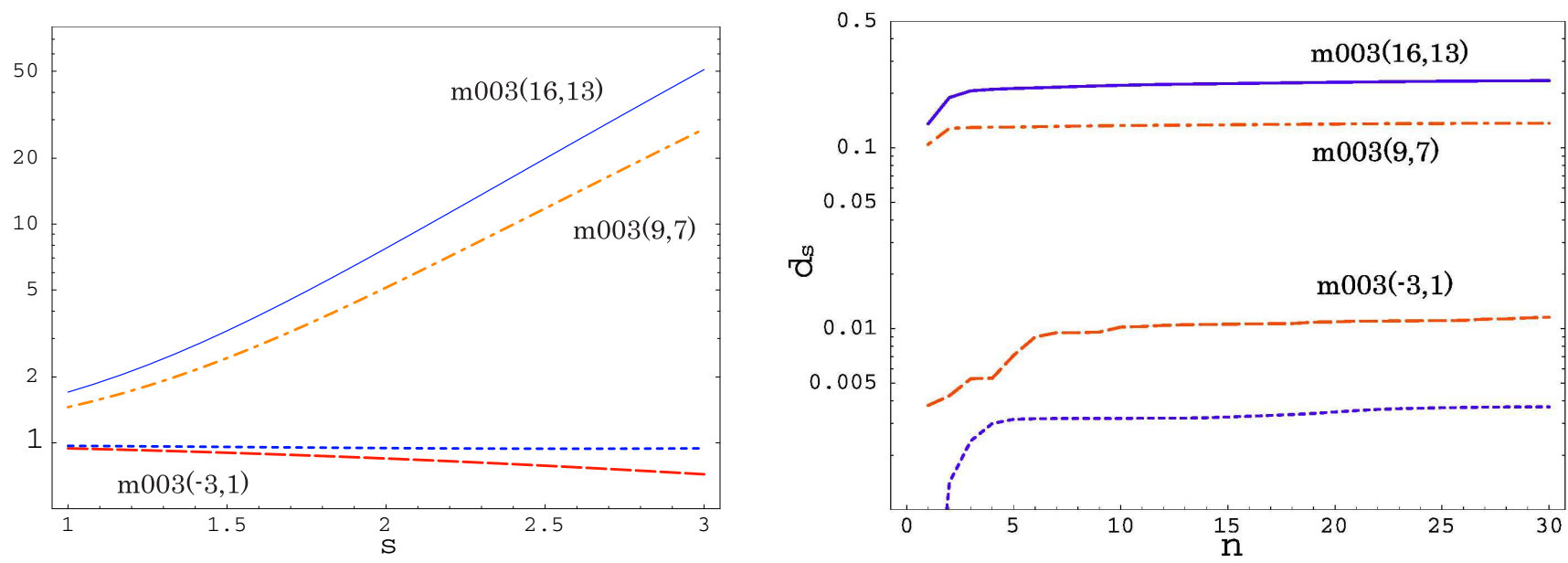

FIG. 11. Spectrum measurements of the geometry. The figure in the left shows $\Delta(s)=\zeta(s) / \zeta_{w}(s)$ for 3 examples of CH 3 -manifolds ,m003(9,7) (dashed-dotted curve), m003(16,13) (solid curve), m003(-3,1) (dashed curve) and m003(16,13) where the shortest periodic orbit is removed (dotted curve). The figure in the right shows $d_{s}(n)=\frac{1}{2} \sum_{i=1}^{n} \ln \frac{1}{2}\left(k_{i} / k_{w i}+k_{w i} / k_{i}\right)$ for the same examples where $n$ is the cutoff number in the summation.

Next, we consider the spectral distance $d_{s}$ proposed by Seriu which measures the degree of semi-classical quantum decoherence between two universes having one massless scalar field [30],

$$
d_{s}[M, \tilde{M}] \equiv \frac{1}{2} \sum_{i=1}^{\infty} \ln \frac{1}{2}\left(\frac{k_{i}}{\tilde{k}_{i}}+\frac{\tilde{k}_{i}}{k_{i}}\right)
$$

where $k_{i}^{2}$ and $\tilde{k}_{i}^{2}$ are the eigenvalues of the Laplace-Beltrami operator on a compact n-manifold $M$ and $\tilde{M}$, respectively. Here we choose eigenvalues $k_{w i}^{2}$ as $\tilde{k}_{i}^{2}$. In practice we introduce a cutoff in the summation. It is numerically found that the summation converges rapidly for the 3 examples, namely, m003(-3,1), m003(9,7) and m003(16,13). The contribution of the first several terms dominates the summation (figure 11). The result implies that a universe having a spatial geometry m003(9,7) or m003(16,13) semiclassically decoheres with a universe having a spatial geometry m003(-3,1) (figure 12). 

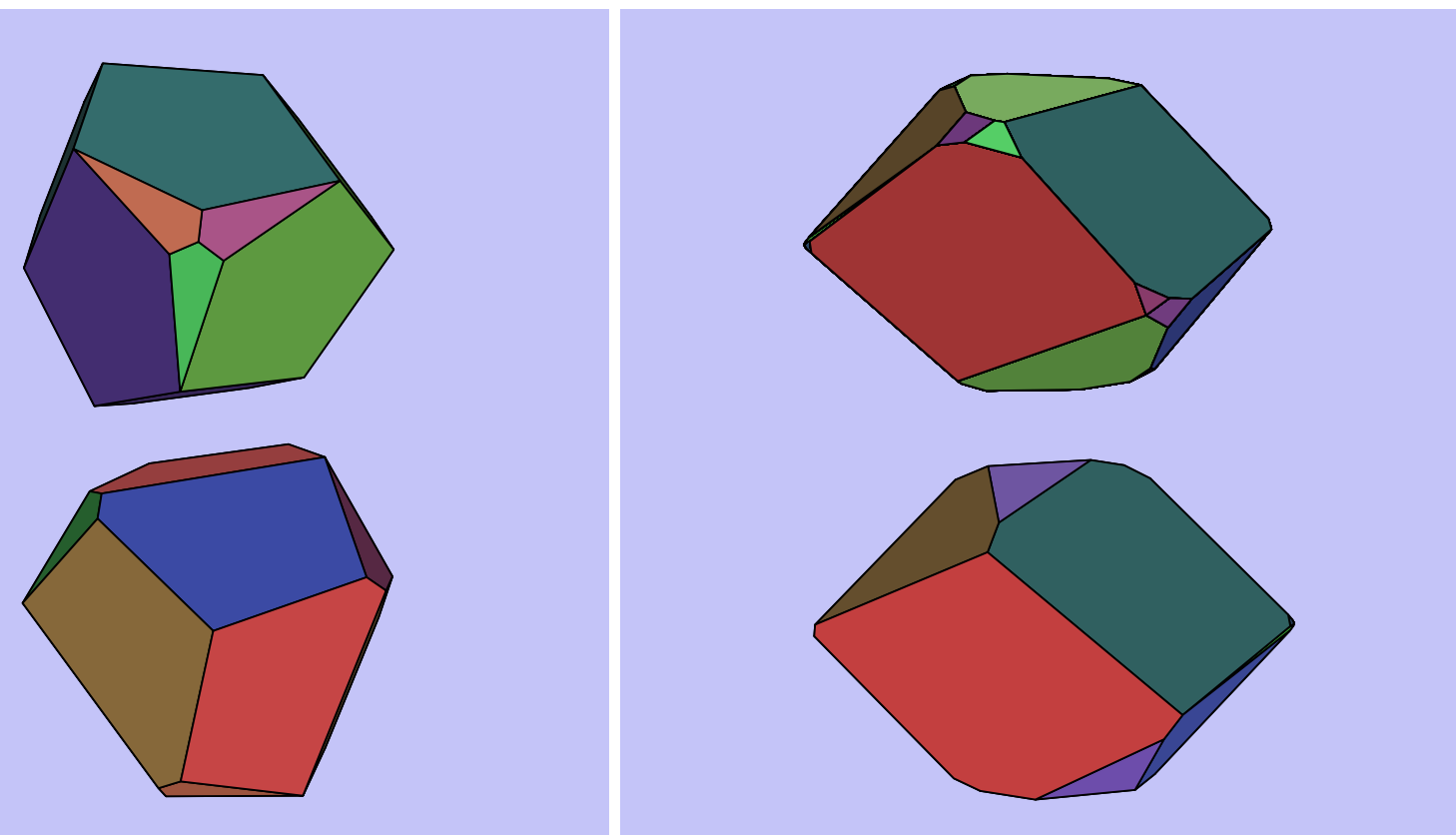

FIG. 12. Plots of a Dirichlet domain of the Weeks manifold m003(-3,1) (left) and that of m003(16,13) (right) viewed from two opposite directions in the Klein coordinates. The Dirichlet domain of m003(16,13) is quite similar to that of the original cusped manifold m003 (see figure 2). m003(16,13) has a "thin" part which is similar to the neibourhood of a cusp. The colors on the faces correspond to the identification maps.

\section{SUMMARY}

In this paper, the length spectra and low-lying eigenvalue spectra of the Laplace-Beltrami operator on small $\mathrm{CH}$ 3-manifolds have been numerically investigated. $\mathrm{CH} 3$-manifolds are relevant to a various kinds of physical systems. For instance, computing the CMB anisotropy in $\mathrm{CH}$ models is one of the key issue which have been investigated for several models 331,29, 32, 33. In order to fully understand to what extent $\mathrm{CH}$ models are constrained by the observations, it is necessary to explore the property of low-lying eigenvalues and eigenmodes for a large number of manifolds (or orbifolds).

First, The length spectra for a total of $308 \mathrm{CH} 3$-manifolds (volume less than 3) have been successfully computed using the SnapPea kernel up to the length $l=7.0$. The asymptotic behavior in the classical staircase is found to be consistent with the known analytical formula which does not depend on the topology or symmetry of the manifold. Regarding the symmetry of the length spectra, it is well known that the arithmetic structure breaks a generic feature: the locally averaged multiplicity number grows exponentially as $l$ is increased. However, the exponential behavior in the multiplicity number which has been noticed for a non-arithmetic 3-orbifold has been confirmed for the smallest five examples of non-arithmetic 3-manifolds, though the rate is smaller than that for the arithmetic manifolds. This may be related to the "hidden symmetry" which is the symmetry of finite sheeted covers of the manifold (which can be tessellated by the copies of the fundamental domain of the manifold) but not of the manifold itself.

Next, the trace formula has been applied to these 3-manifolds for computing low-lying eigenvalues using the length spectra (POSM). Consistency with those obtained by the DBEM has been confirmed for several manifolds. It is numerically found that these manifolds do not have any supercurvature modes. It seems that the manifolds which supports supercurvature modes are either having large volume or large diameter. In order to confirm this, further investigation of eigenvalues for cusped manifolds is necessary.

Thirdly, the first eigenvalues are compared to a various diffeomorphism-invariant quantities, namely, diameter, volume and the shortest length of the periodic orbits. The numerical results imply the existence of much shaper bounds for the first eigenvalues in terms of diameter. Some fitting formulae have been introduced and their validity has been checked. We have seen that $\mathrm{CH}$ 3-manifolds can be roughly divided into two categories: "slightly anisotropic" and "almost anisotropic" ones. The former has not any very short periodic orbits while the latter has. For example, manifolds which are very similar to the original cusped manifold are belonging to the latter category. It is found that 
the deviation of the spectrum from the Weyl asymptotic formula for these manifolds is conspicuous even for manifolds with small volume.

Finally, the global "anisotropy" in the spatial geometry has been measured by $\zeta$-function and the spectral distance for 3 examples of $\mathrm{CH} 3$-manifolds. These measurements give a clear indication of the presence of a "thin" part in the manifold in terms of eigenvalue spectra. In other words, the physical quantities (the angular power spectra in the $\mathrm{CMB}$ or decoherence between two universes) are greatly affected by the globally anisotropic structure in the spatial geometry.

\section{ACKNOWLEDGMENTS}

I would like to thank Jeff Weeks for his extensive advice on the use of SnapPea and excellent explanation on geometry and topology of $\mathrm{CH}$ spaces and Ralph Aurich for his informative comments on the trace formula. I would also like to thank Ian Agol for pointing out some mistakes in the manuscript. The numerical computation in this work was carried out at the Data Processing Center in Kyoto University and Yukawa Institute Computer Facility. K.T. Inoue is supported by JSPS Research Fellowships for Young Scientists, and this work is supported partially by Grant-in-Aid for Scientific Research Fund (No.9809834).

[1] Brooks R 1992 Topology '90 Ohio. State Univ. Math. Res. Inst. Publ., 161 (Berlin)

[2] Bohigas O 1991 Proceedings of the 1989 Les Houches School on Chaos and Quantum Physics ed Giannoni A et al (Amsterdam: Elsevier)

[3] Balazs N L and Voros A 1986 Phys. Rep. 1433109

[4] Aurich R and Steiner F 1989 Physica D 39169

[5] Aurich R and Steiner F 1990 Physica D 43155

[6] Aurich R and Steiner F 1991 Physica D 48445

[7] Bolte J Steil G and Steiner F 1992 Phys. Rev. Lett. 69152188

[8] Aurich R and Steiner F 1993 Physica D 64185

[9] Aurich R and Steiner F 1995 Phys. Rev. E 5154173

[10] Aurich R and Marklof J 1996 Physica D 92101

[11] Gutzwiller M C 1990 Chaos in Classical and Quantum Mechanics (Springer: New York)

[12] Selberg A 1956 J. Indian. Math. Soc. 2049

[13] Hejhal D A 1991 International Symposium in Memory of Hua Loo-Keng ed Gong S Lu Q and Yang L (New York: Science)

[14] Grunewald F and Huntebrinker W 1996 Experimental Mathematics 51

[15] Inoue K T 1999 Class.Quant.Grav. 163071

[16] Inoue K T 2000 Phys. Rev. D 62103001

[17] Cornish N J and Spergel D N 1999 preprint math.DG/9906017

[18] Weeks J R SnapPea: a Computer Program for Creating and Studying Hyperbolic 3-manifolds, available at: http://www.northnet.org/weeks

[19] Thurston W P 1982 Bulliten (New Series) of the American Math Society 63357

[20] Matveev S V and Fomenko A T 1988 Uspekhi Mat. Nauk 4313

[21] Hodgson C D and Weeks J R 1994 Experimental Math. 34261

[22] Margulis G 1969 Funct. Anal. Appl.3 335

[23] Aurich R and Steiner F 1988 Physica D 32451

[24] Aurich R, Scheffler F and Steiner F 1995 Phys. Rev. E 5154173

[25] Coulson D, Goodman O A, Hodgson C D and Neumann W D 2000 Experimental Math. 91127

[26] Cheng X and Zhou D 1995 Hokkaido Mathematical J. 24453

[27] Lu Y G 1995 Bol. Un. Mat. Ital. A (7) 93461

[28] Cheng S-Y 1975 Math. Z. 143289

[29] Inoue K T 2000 Mon. Not. R. Astron. Soc. 3144 L21

[30] Seriu M 1996 Phys. Rev. D 53126902

[31] Bond J. R., Pogosyan D. and Souradeep T. 2000 Phys. Rev. D. 62043006

[32] Aurich R 1999 Astrophys. J. 524497

[33] Cornish N J and Spergel D N 2000 Phys. Rev. D 62087304 


\section{APPENDIX A:}

For a given Dirichlet domain $D$, SnapPea computes

1. Neighboring copies of $D$ (tiles) recursively and stores the corresponding elements $g$ of the discrete isometry group $\Gamma$. If it has already been found out, it is discarded. The computation proceeds until for all the neighborhoods of $g D, d(x, h x)>\cosh ^{-1}(\cosh R \cosh l / 2)$ satisfies where $h D^{\prime}$ s are neighborhoods of $g D(R$ is the spine radius and $h$ is an element of $\Gamma$ ). Since there is no $g$ other than identity where all $h^{\prime}$ s satisfy $d(x, h x) \geq d(x, g x)$, this algorithm will not miss any tiles $g D$ where $d(x, g x)<2 \cosh ^{-1}(\cosh R \cosh l / 2)$.

2. A list of geodesics for all $g^{\prime} s=\{g\}$ where 1:the real part of length is not zero and less than $l$; 2:the distance from $x$ to the geodesic is at most $R$.

3. The conjugacy class $g^{\prime}=h g h^{-1}$ or its inverse for each $g$ where $h$ is an element of $\{g\}$. If an identical complex length is found, the complex length which corresponds to $g^{\prime}$ is omitted from the list of geodesics. If the complex length of $g$ is conjugate to that of $g^{-1}$, the geodesic is topologically a mirrored interval, otherwise it is a circle.

4. Multiplicity of geodesics. If a pair of geodesics with two complex lengths being identical within an error range is found, the multiplicity number is increased by one.

\section{APPENDIX B:}

Suppose a $\mathrm{CH}$ manifold $M$ which resembles the original cusped manifold $M_{c}$ with one cusp. Let us divide $M_{c}$ into two parts, the neighbourhood of a cusped point $K_{c}$, and the complementary part $K_{c 0}$. Similarly, one can divide $M$ into $K$ and $K_{0}$ where $K_{c 0} \approx K_{0}$ and $K$ corresponds to a "thin" part. Since the neighbourhood of a cusp is represented as a "chimney" (but having infinite length) in the upper half space coordinates $\left(x_{1}, x_{2}, x_{3}\right), K$ can be well approximated by an elongated box defined by $\left(-\Delta x / 2 \leq x_{1} \leq \Delta x / 2,-\Delta x / 2 \leq x_{2} \leq \Delta x / 2, x_{30} \leq x_{3} \leq x_{31}\right)$. Then the physical length(=diameter) $\tilde{d}$ of $K$ in the direction $x_{3}$ is given by $\tilde{d}=\ln \left(x_{31} / x_{30}\right)$. On the other hand, the volume $\tilde{v}$ of $K$ satisfies

$$
\tilde{v}=\frac{(\Delta x)^{2}}{2}\left(\frac{1}{\left(x_{30}\right)^{2}}-\frac{1}{\left(x_{31}\right)^{2}}\right)
$$

which gives the ratio of the volume $\tilde{v}$ to the volume of $\tilde{v}_{\infty}$ of $K_{c}, \tilde{v} / \tilde{v}_{\infty}=1-\left(x_{30}\right)^{2} /\left(x_{31}\right)^{2}=1-\exp (-2 \tilde{d})$. If we approximate the diameter $d$ of $M$ as $d=\tilde{d}+d_{0}$ where $d_{0}$ is the diameter of $K_{0}$, then we finally have the ratio of the volume $v=v_{0}+\tilde{v}$ of $M$ to the volume $v_{c}=v_{0}+\tilde{v}_{\infty}$ of $M_{c}$,

$$
\frac{v}{v_{c}}=1-\frac{\exp \left(-2\left(d-d_{o}\right)\right)}{\delta+1}, \quad \delta \equiv v_{0} / \tilde{v}_{\infty}
$$

where $v_{0}$ denotes the volume of $K_{0}$. 


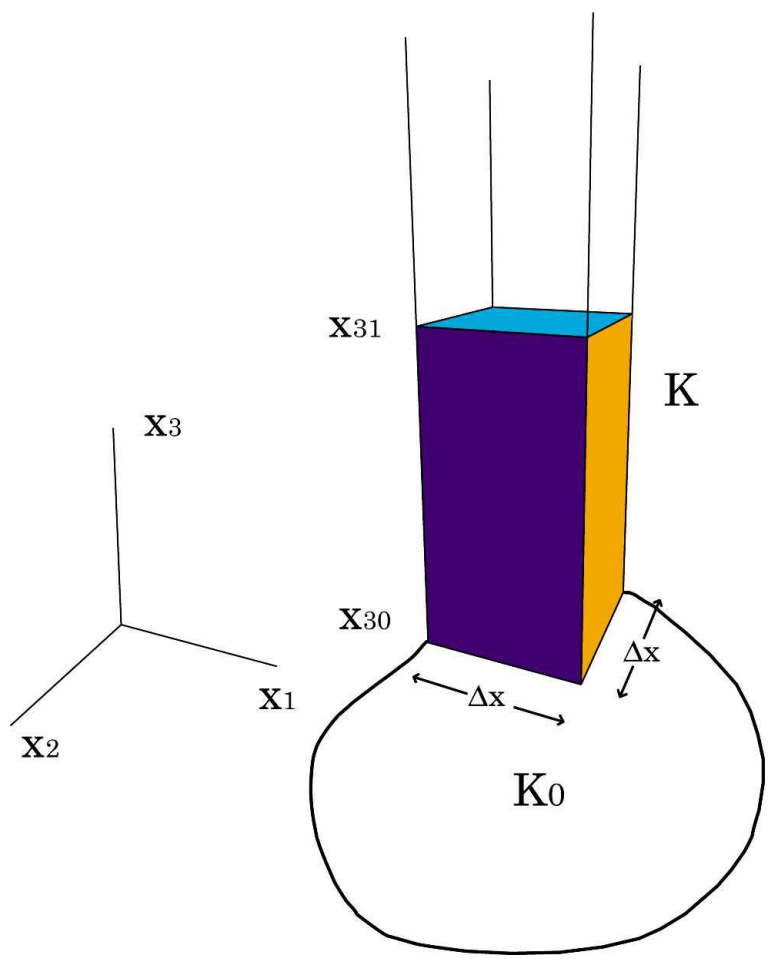

FIG. 13. Estimate of the volume of a "thin" part $K$ in a manifold $M$ in the upper half space. 
This figure "Nm003p9q7.gif" is available in "gif" format from: http://arxiv.org/ps/math-ph/0011012v2 
This figure "Nm003p16q13.gif" is available in "gif" format from: http://arxiv.org/ps/math-ph/0011012v2 